\title{
Intravenous Maintenance Fluid Therapy in the Pediatric Acute and Critical Care Setting: A European Practice Survey. Intravenous Maintenance Fluids in Pediatric Practice: A European Survey
}

\section{Claire Morice}

Geneva University Hospitals: Hopitaux Universitaires Geneve

Fahad Alsohime

King Saud bin Abdulaziz University for Health Sciences

Huw Mayberry

Alder Hey Children's Hospital

Lyvonne Tume

Alder Hey Children's Hospital

David William Brossier ( $\sim$ brossier-d@chu-caen.fr)

Centre Hospitalier Universitaire de Caen https://orcid.org/0000-0002-2059-9888

\section{Frédéric Valla}

University Hospital Centre Lyon: Hospices Civils de Lyon

ESPNIC IVMFT GROUP (Isabelle Goyer)

CHU Caen: Centre Hospitalier Universitaire de Caen

\section{Research Article}

Keywords: Child, neonate, adolescent, intravenous fluids, in-hospital, balanced solutions.

Posted Date: December 9th, 2021

DOI: https://doi.org/10.21203/rs.3.rs-1124702/v1

License: (c) (i) This work is licensed under a Creative Commons Attribution 4.0 International License. Read Full License 


\section{Abstract}

\section{Background:}

The ideal fluid for intravenous maintenance fluid therapy (IV-MFT) in acutely and critically ill children is controversial and evidence based clinical practice guidelines are lacking. The current prescribing practices remains unknown.

Aim:

We aimed to describe the current practices and choice of pediatric acute care clinicians in prescribing IVMFT in the context of acutely and critically ill children with regards to the amount, tonicity, composition, use of balanced fluid and prescribing strategies in various clinical contexts.

\section{Method:}

A cross-sectional electronic survey was emailed in April-May 2021 to pediatric critical care physicians across European and Middle East countries. The survey instrument was developed by an expert multiprofessional panel within ESPNIC. The survey instrument included their practice of prescribing the IVMFT: indication, amount, tonicity, use of balance solutions \& composition of IV-MFT.

\section{Results:}

154 respondents from 35 European and Middle East countries participated in this survey (response rate $64 \%)$. Respondents were staff physicians or nurse practitioners in charge of critically ill children. They all indicated that they routinely use a predefined formula to prescribe the amount of an IV-MFT. The use of balanced solution was preferred in case of altered serum $\mathrm{Na}$ and $\mathrm{Cl}$ levels or metabolic acidosis. $42 \%$ of responders (65/153) believed that balanced solutions should always be used. In terms of the indication and the composition of IV-MFT prescribed, responses were heterogenous among centers. $70 \%$ of the respondents $(n=107)$ believed there was a gap between the current practice in their unit and what they considered ideal IV-MFT due to the lack of guidelines and inadequate training of health care professionals.

\section{Conclusion:}

Our study showed considerable variability in clinical prescribing practice of IV-MFT in PICUs across Europe and the middle east. There is an urgent need to develop evidence-based guidelines for IV-MFT prescription in acutely and critically ill children.

\section{What Is Known}

- The administration of maintenance intravenous fluid therapy is a standard of care for a lot of hospitalized children 
- Maintenance intravenous fluid therapy prescriptions are often based on Holliday and Segar's historical guidelines even if this practice has been associated with several complications.

\section{what is new:}

- This study showed considerable variability in clinical prescribing practice of intravenous maintenance fluid therapy across Europe and the middle east.

- There is little consensus about the use of intravenous maintenance fluid therapy across acute pediatric practice.

\section{Introduction}

Currently there is little consensus about the use of intravenous (IV) maintenance fluid therapy (IV-MFT) across acute pediatric practice. Maintenance fluid therapy corresponds to the "volume of fluid required to meet daily metabolic needs, such as normal water and electrolyte losses, and maintain homeostasis." (1) and should be distinguished from resuscitation and replacement/redistribution fluid therapy. There has been much debate about which IV-MFT solution to use and the amount to give. (2-8)

Fluids are considered iso-, hyper-, or hypo-tonic if their tonicity (also called fluid effective osmolarity) is almost, above, or under plasma tonicity respectively. Balanced fluids are characterized by their chloride content, which is close to plasma chloride content, its use has been increasingly adopted as the fluid of choice as it causes less acidosis and electrolyte disturbance than chloride-rich solutions. In these solutions, part of the chloride anion is replaced by organic anions (such as malate, acetate or gluconate) which maintain the anion/cation balance. $(9,10)$

In the context of developing new European guidelines, on behalf of ESPNIC (The European Society of Pediatric and Neonatal Intensive Care) it is important to understand current practice across Europe. Previous surveys on IV-MFT have focused mainly on the tonicity of fluids prescribed, with a preference for hypotonic fluids in the early 2010 s $(11,12)$ and shifting towards a preference for isotonic fluids approaching and during 2020 (13). Historically, the amount of IV-MFT has been dictated by the Holliday and Segar formula produced in 1957 (14). The aim of our study was to describe the current European practice of prescribing the IV-MFT, not only on tonicity, but also of the use of balanced fluids, amount prescribed, fluid composition (i.e., glucose, potassium, calcium, potassium, magnesium, and micronutrient content) and IV-MFT prescription strategy within different pediatric clinical contexts for children admitted to general pediatric wards (acutely ill children) and pediatric intensive care units (PICU) (critically ill children).

\section{Methods}

\section{Study design and method:}


We conducted a cross-sectional electronic, survey of healthcare workers (HCW) prescribing IV MFT for acutely \& critically ill pediatric patients.

The sampling frame included all HCWs working in the PICUs within the ESPNIC Network.

\section{Survey instrument development and content}

The survey was developed in English by a multi-professional European ESPNIC group, leading the project to develop ESPNIC guidelines on IV-MFT in acutely and critically ill children (Supplemental Digital Content 1). The scope of the survey included term neonates ( $>37$ weeks gestational age) and children up to 17 years of age. It was made clear that fluid bolus for resuscitation, replacement/redistribution fluid therapy and intraoperative fluid therapy were outside the scope of the survey. Following a review of the literature and previous surveys, a new 27-item-cross-sectional survey was constructed and reviewed by an expert panel of 6 members of the ESPNIC IV-MFT group for content validity. This was tested for face validity on four physicians, and following this, minor changes were made to the wording to improve clarity.

The final 27-questions survey included the demographic characteristics of respondents, then questions were divided into five different sections, which correspond to the five main domains of the future ESPNIC IV-MFT guidelines: i) indications for IV MFT, ii) amount of IV-MFT, iii) tonicity of IV-MFT - iv) balanced solutions, and v) composition of IV MFT (glucose, K, Mg, Ca, P, micronutrients). [Survey instrument Electronic Supplementary File 1]. The questions were elaborated to evaluate their practice and the factors that affect their decisions when prescribing IV-MFT. Different common clinical scenarios such as gastroenteritis, status epilepticus, bronchiolitis and post-appendectomy in different age groups (7-day old neonate, 5 months old infant and 12 years old adolescent) were presented to the respondents.

Responders had to answer questions according to 6 Points-Likert scale, or 0 to 10 rating scale or multiple choices.

\section{Data collection}

The electronic survey was disseminated online in April-May2021 within the ESPNIC network, via SurveyMonkey ${ }^{\circledR}$ software (SanMateo, California, USA). The survey began with an invitation letter with instructions clarifying the scope of the survey and how to answer it, specifying answers should describe local current practices rather than ideal practices. Completion of the survey implied voluntary consent to participate in the research. Fourteen members of the ESPNIC IV-MFT guideline group volunteered and acted as references in their European region with their respective networks. We specified only one response per center. In view of the ESPNIC network, we anticipated answers from 100 different units. We aimed for a response rate above $60 \%$, so a maximum of two reminder emails were sent to nonresponding centers. To avoid bias with one country dominating the survey, no reminders were sent within a country if already more than 15 responses were received.

\section{Data analysis}


Data was exported from a CSV file into Microsoft Excel for further checking and descriptive analysis. Questionnaires more than $10 \%$ incomplete were excluded from the study. Percentages were used to summarize categorical data. We used a summative score to summarize the results from Likert scale questions for each participant. Continuous variables were presented as median and inter-quartiles (IQR) or mean and standard deviation (SD) depending on the variables' distribution and frequency and proportions for categorical variables. Comparisons between both groups were made by Paired $t$ test or a paired samples Wilcoxon test according to the distribution for continuous variables, as appropriate. Results were considered statistically significant at $\mathrm{p}$ value less than 0.05 . and two tailed tests were used Tests were performed using BiostaTGV.

Ethical approval was obtained from Caen-France institutional review board (reference number 2474) for the study.

\section{Results}

Full answers are available in supplementary electronic material (Supplemental Digital Content 2)

\section{Participants' characteristics}

One hundred and fifty-four responses were received from 35 European and Middles Eastern countries of the 240 PICUs contacted in 43 countries (response rate 64\%). The characteristics of the respondents are detailed in Table 1 and Figure 1.

\section{Indications for IV maintenance fluids}

Responses regarding the indications for IV-MFT were heterogenous among centers and respondents. The patient's condition was the main criterion to prescribe IV-MFT as shown in table 2 . The respondents indicated that they would always prescribe IV-MFT rather than prescribing enteral hydration/nutrition in case of severe DKA, 84\% (128/154), post-abdominal surgeries, 74\% (113/154). Regarding the other situations, practices varied between respondents and none appeared preponderant (table 2), expected regarding bronchiolitis, a situation in which a slight majority of the respondents $(54 \%(82 / 154)$ considered never to rarely prescribe IV-MFT.

\section{Amount of IV fluids}

Fluid balance monitoring was considered important by all the respondents in the management of critically ill children (mean score 10/10 \pm 0.8 ) and in acutely ill children (mean score 8/10 \pm 1.9 ). All respondents indicated they routinely use the following formulas to prescribe the amount of an IV-MFT, most commonly Holliday-Segar $76 \%$ (117/154), followed by Oh $23 \%(35 / 154)$ then Adelman and Solhaugh $15 \%$ (23/154). However, a fluid restriction strategy was applied in some cases such as children with cardiac condition (87\% of the respondents, $130 / 154)$, children following cardiac surgery $(86 \%$ of respondents, $113 / 154)$, children with renal failure $(78 \%, 118 / 154)$ and in children on invasive ventilation 
(56\% of respondents, $84 / 154$ ), (Figure 2 ). Regarding the calculation of total fluid intake, the fluids included in the total fluid intake are shown in figure 3.

\section{Type of solution/isotonic}

The majority of the respondents rated the importance of the use of isotonic IV solutions for critically ill children very highly (mean score $9 / 10 \pm 1.9$ ) and acutely ill children (mean score $8 / 10 \pm 2.0$ ). The fluids selected by the respondents for each scenario are shown in Table 3.

\section{Balanced solutions}

Prescribing a balanced fluid as IV-MFT for the critically and acutely ill pediatric patients was considered very important by the respondents (mean score $8 / 10 \pm 2.4$ and 7/10 \pm 2.6 respectively). The criteria for selecting the balanced IV solution as IV-MFT were consistent among the respondents. Most would prescribe balanced IV solutions in cases of altered serum chloride levels $(78 \%, 120 / 154)$, metabolic acidosis $(75 \%, 116 / 154)$, altered serum Na levels $(62 \%, 96 / 154)$, according to the child's underlying clinical condition $(60 \%, 92 / 154)$.

\section{Fluid composition}

The median age that glucose was no longer perceived to be required to be added to the IV-MFT was 12 years (IQR 4,75 - 16). 46\% (70/154) of respondents indicated they always prescribed glucose with the IVMFT, and $52 \%(80 / 154)$ that they often prescribed it. For potassium supplementation in IV-MFT, $25 \%$ (38/154) indicated they always prescribed it while $73 \%(112 / 154)$ often prescribed it. Calcium was always prescribed by $9 \%(14 / 154)$ while $63 \%(96 / 154)$ often prescribed it and $28 \%(43 / 154)$ rarely prescribed it. For the other elements such as phosphate, magnesium, trace elements and vitamins, these were rarely/never prescribed by $51 \%(77 / 154)$ of participants (see table 4 for further details).

In terms of IV-MFT practices, $70 \%$ (107/153) of responders believed there was a gap between their current practice and what they considered ideal IV-MFT practice, especially outside the PICU setting. The main reasons for this were believed to be the lack of guidelines (32\% 49/153) and inadequate training of health care professionals $(26 \% 39 / 153)$ and lack of access to ready to use solutions. There was a wide range of "ready to use" IV-MFT solutions used in each hospital (in PICU and general pediatric wards), with only $4 \%$ of respondents $(6 / 153)$ not having access to ready to use IV fluid solutions $14 \%(22 / 153)$.

\section{Discussion}

To our knowledge, this is the first European survey that has described multiple aspects of IV-MFT in acute pediatric and intensive care. Our results show a wide variation in practice in IV-MFT in children across Europe. IV-MFT should be considered like any other drug, with side effects and consequences (1). The indications for IV-MFT are varied. The Enhanced Recovery After Surgery (ERAS) protocol recommends avoiding prolonged IV-MFT by starting enteral nutrition/fluids early (11). Whenever it is possible, the IV- 
MFT should not be the first line of hydration as it is associated with greater potential for loss of nutritional status and iatrogenic electrolyte disturbances. (12)

In 1957, Holliday and Segar published a formula to guide the prescribing of pediatric IV-MFT volume. We found that this formula still dominates practice, despite the limitations of this original paper, which was based on the energy requirements of healthy, well hydrated children (14). We still have little definitive evidence that Holliday and Segar is the optimum formula (15). Indeed, there are many situations where it was felt prudent to restrict fluids beyond this standard calculation. Our survey showed that IV-MFT fluid was commonly restricted in children with cardiac conditions, renal failure, invasive mechanical ventilation and often in

children following cardiac surgery, with no respondents reporting exceeding the standard maintenance fluid volumes.

The majority of the respondents reported that they included most of the fluids received by the patients while calculating the total fluid intake and daily fluid balance (enteral \& parenteral fluids).

Underhydration has rarely been reported in the literature, in contrast to the impact of overhydration. The frequency and adverse effects of fluid overload is increasingly reported in critically ill children leading to longer duration of mechanical ventilation, the need for renal replacement therapy and longer duration of ICU stay. (16) This is due to multiple factors, one being that critically ill children may have increased levels of secreted anti-diuretic hormone (ADH) to compensate for the initial hypovolemia, which predisposes them to fluid retention and hyponatremia (17).

This study highlighted that most respondents have chosen to prescribe the isotonic solution for maintenance intravenous fluid for the critically and acutely ill children. This finding is aligned with the latest recommendation of the American Academy of Pediatrics (AAP) Clinical Practice Guideline which recommend the use of isotonic fluid therapy instead of hypotonic fluid therapy. (18)

This recommendation has markedly changed the prescribing of IV-MFT practices in children toward isotonic fluid therapy (19). The aim of this recommendation was to prevent adverse events associated with iatrogenic hyponatremia and acute or permanent neurological impairment associated with the administration of hypotonic solutions in contrast to isotonic solutions. (18). Moreover, fatal hyponatremia has been reported in children receiving hypotonic fluid therapy. $(20,21)$. On the other hand, children receiving isotonic fluid therapy have an increased risk for hypernatremia, which has previously been associated with increased risk of mortality if left untreated. (19)

The results of our study are consistent with recent observational studies, indicating that the unbalanced crystalloids are the most used maintenance fluids, whereas factors contributing to the decision of prescribing balanced salt solutions were mainly related to the serum chloride level, presence of metabolic acidosis and patient's clinical condition respectively. $(5,22,23)$ 
Debates have focused on whether chloride rich solutions worsen patient's outcome through the increased risk of hyperchloremic acidosis and whether the physiologically balanced solutions may improve or ameliorate these outcomes. Notably, potential side effects related to saline-chloride use have been identified, including hyperchloremic acidosis, nephrotoxicity, coagulopathy, gastrointestinal dysfunction and increased mortality. studies have shown that hyperchloremia produces consequent risks of coagulopathy, renal vasoconstriction, heightened inflammatory response in the kidneys through the release of eicosanoids and results in reduced renal cortical tissue perfusion and has been associated with higher incidence of acute kidney injury. (23-26) In adults, several studies have reported a higher incidence of metabolic acidosis and hyperchloremia in patients who received saline compared with balanced solutions. $(27,28)$

Whereas for the physiologically balanced salt solutions such as $\mathrm{RL}$, the lactate in $\mathrm{RL}$ gets converted to bicarbonate via gluconeogenesis and oxidation not only in the liver but also in the kidneys and can improve $\mathrm{pH}$ and may ameliorate this harm associated with the chloride-rich solutions. (29)

However, there is a lack of robust evidence to be able to recommend the use of one isotonic crystalloid over another one in children. Although some societies/organizations such as the North American Society for Pediatric Gastroenterology (2018), Hepatology and Nutrition and the WHO advocates Ringer lactate as preferred fluid for fluid therapy in cases of acute pancreatitis and for correction of severe diarrheal dehydration respectively. $(29,30)$

Although the understanding of metabolic response to critical illness has evolved over the last decade, there is still huge variability in daily practice around the ideal composition of IV-MFT. $(31,32)$. Glucose is the preferential energy substrate during critical illness and a lack of glucose supply leads to ketogenesis and neurological effects (33), and most respondents still prescribed glucose in IV-MFT in young children. However, the age at which glucose was no longer prescribed in fluids was very heterogeneous.

The addition of electrolytes to IV-MFT was highly variable, probably due to the lack of recommendations to guide the clinicians. The AAP recommends using solutions with appropriate levels of potassium chloride, most commonly $2 \mathrm{mmol}$ of potassium per $100 \mathrm{kcal}$ metabolized (34). However, despite this recommendation most ready to use maintenance IV fluid solutions do not meet these recommendations, (e.g., Ringers Lactate contains $0.4 \mathrm{mmol} / \mathrm{kg} / \mathrm{L}$ ). The practice of adding micronutrients to IV-MFT was also rare. A recent systematic review of micronutrients studies in critically ill children revealed that micronutrients should be provided in sufficient amount to the critically ill pediatric patients, but there was insufficient data to recommend routine supplementation of micronutrients at higher doses during the critical illness. (34)

\section{Limitations of the Study}

This study has some limitations, inherent to its design. The self-report nature of the survey risks bias, and may reflect individual views rather than actual practice, and selection bias, caused by the voluntary nature of the survey, may have resulted in clinicians with a greater interest in the topic answering. 
Moreover, as PICU intensivist or anesthetists were surveyed, the accuracy of the practice on general pediatric wards may be less reliable. However, our response rate is good, improving the reliability of the survey and it is the first survey to engage with clinicians across Europe and the middle east around broader practices around IV-MFT in children.

\section{Conclusions}

Our study showed considerable variability in pediatric clinical practice around IV-MFT. There is an urgent need to conduct more robust research and develop evidence-based guidelines for IV-MFT in critically/acutely ill children to guide clinical practice. This survey may also be used after the dissemination of future guidelines to assess the change in practice.

\section{Abbreviations}

ESPNIC European Society of Pediatric and Neonatal Intensive Care

IV-MFT Intravenous maintenance fluid therapy

PICU Pediatric intensive care unit

IV Intravenous

HCW Healthcare worker

NICU Neonatal intensive care unit

DKA Diabetes ketoacidosis

ARDS Acute respiratory distress syndrome

ERAS Enhanced Recovery After Surgery

AAP American academy of pediatrics

$\mathrm{RL} \quad$ Ringer's lactate

NS Normal saline

\section{Declarations}

\section{Acknowledgment}

We acknowledge with gratitude those respected colleagues who were involved in the distribution of the survey within their country and region: Sanja Simic and Dejan Milojevic (Serbia), Jeppe Sylvest Angaard 
Nielsen (Danemark), Mari-Liis Ilmoja (Estonia), Josko Markic (Croatia), Rachel Elizabeth Grech (Malta), Reinis Balmaks (Latvia).

Funding: This work has not been funded.

Conflicts of interest/Competing interests: FVV declares consulting fees received from BAXTER. For the remaining authors none were declared.

Availability of data and material: Raw data are submitted as supplemental material

Code availability: Not Applicable

Authors' contributions: FVV conceived the study; CM, FVV, DB and LT developed the study protocol CM, FVV. LT, DB, HM, FA developed and piloted the survey tool; CM was responsible for data collection; CM, FVV, LT, DB, HM, FA analysed the results and drafted the manuscript

Ethics approval: Our protocol was analysed within the Research Ethics Committee (CLERS) and was approved on May 2021. Due to the nature of the study, the Institutional Review Board waived the need for informed consent.

Consent to participate: Not Applicable

Consent for publication: Not Applicable

\section{References}

1. Malbrain, M.L.N.G., Langer, T., Annane, D. et al (2000) Intravenous fluid therapy in the perioperative and critical care setting: Executive summary of the International Fluid Academy (IFA). Ann. Intensive Care 10:64

2. Allen $\mathrm{CH}$, Goldman RD, Bhatt $\mathrm{S}$, et al (2016) A randomized trial of Plasma-Lyte A and $0.9 \%$ sodium chloride in acute pediatric gastroenteritis. BMC Pediatr 16:117

3. Mahajan V, Sajan SS, Sharma A, et al (2012) Ringers lactate vs normal saline for children with acute diarrhea and severe dehydration-a double blind randomized controlled trial. Indian Pediatr 49:963968

4. Choong K, Kho ME, Menon K, Bohn D (2006) Hypotonic versus isotonic saline in hospitalised children: a systematic review. Arch Dis Child 91(10):828-835

5. Foster BA, Tom D, Hill V: Hypotonic versus isotonic fluids in hospitalized children: a systematic review and meta-analysis. J Pediatr 2014,165(01):163-169.e2

6. Chowdhury AH, Cox EF, Francis ST, Lobo DN (2012) A randomized, controlled, double-blind crossover study on the effects of 2-L infusions of $0.9 \%$ saline and Plasma-Lyte ${ }^{\circledR} 148$ on renal blood flow velocity and renal cortical tissue perfusion in healthy volunteers. Ann Surg 256(01):18-24

7. Hatherill M (2004) Rubbing salt in the wound. Arch Dis Child 89:414-18 
8. Mann NP (2004) What routine intravenous maintenance fluids should be used? Arch Dis Child 89:411

9. Semler MW, Kellum JA (2019) Balanced Crystalloid Solutions. Am J Respir Crit Care Med 199(8):952960

10. Cecconi M, Hofer C, Teboul JL, et al (2015) Fluid challenges in intensive care: the FENICE study: a global inception cohort study. Intensive Care Med 41:1529-1537

11. Rove KO, Edney JC, Brockel MA: Enhanced recovery after surgery in children: Promising, evidencebased multidisciplinary care. Paediatr Anaesth 2018,28(6):482-492

12. Oakley E, Borland M, Neutze J, et al (2013) Paediatric Research in Emergency Departments International Collaborative (PREDICT). Nasogastric hydration versus intravenous hydration for infants with bronchiolitis: a randomised trial. Lancet Respir Med 1(2):113-120

13. Intravenous fluid therapy in children and young people in hospital. London: National Institute for Health and Care Excellence (UK), 2020 Jun 11. PMID: 33108143

14. Holliday, M., Segar W (1957) The maintenance need for water in parenteral fluid therapy Malcolm A. Pediatrics 19(5):823-832

15. Holliday MA, Ray PE, Friedman AL (2007) Fluid therapy for children: facts, fashions and questions. Arch Dis Child 92(6):546-550

16. Alobaidi R, Morgan C, Basu RK, et al (2018) Association Between Fluid Balance and Outcomes in Critically III Children: A Systematic Review and Meta-analysis. JAMA Pediatr 1,172(3):257-268

17. Moritz ML, Ayus JC (2005) Preventing neurological complications from dysnatremias in children. Pediatr Nephrol 20:1687-1700

18. Feld L, Neuspiel D, Fosteret B et al (2018) Clinical Practice Guideline: Maintenance Intravenous Fluids in Children. Pediatrics 142(6): e20183083

19. Lehtiranta S, Honkila M, Kallio M et al (2021) Risk of Electrolyte Disorders in Acutely III Children Receiving Commercially Available Plasmalike Isotonic Fluids: A Randomized Clinical Trial. JAMA pediatrics 175(1): 28-35

20. Grissinger M (2013): Hyponatremia and death in healthy children from plain dextrose and hypotonic saline solutions after surgery. P T 38(7):364-388

21. Arieff Al, Ayus JC, Fraser CL (1992) Hyponatraemia and death or permanent brain damage in healthy children. BMJ 304(6836):1218-1222

22. Williams V, Jayashree M, Nallasamy K, et al (2020) $0.9 \%$ saline versus Plasma-Lyte as initial fluid in children with diabetic ketoacidosis (SPinK trial): a double-blind randomized controlled trial. Crit Care 2,24(1):1

23. Bulfon AF, Alomani HL, Anton $\mathrm{N}$ et al (2019) Intravenous Fluid Prescription Practices in Critically III Children: A Shift in Focus from Natremia to Chloremia? J Pediatr Intensive Care 8(4):218-225

24. Eisenhut M (2006) Causes and effects of hyperchloremic acidosis. Crit Care 10(3):413 
25. Kellum JA, Song M, Venkataraman R (2004) Effects of hyperchloremic acidosis on arterial pressure and circulating inflammatory molecules in experimental sepsis. Chest 125:243-248

26. Nashat FS, Tappin JW, Wilcox CS (1976) The renal blood flow and the glomerular filtration rate of anaesthetized dogs during acute changes in plasma sodium concentration. J Physiol 256:731-745

27. Young JB, Utter GH, Schermer CR, et al (2014) Saline versus Plasma-Lyte A in initial resuscitation of trauma patients: a randomized trial. Ann Surg 259:255-262

28. Mahler SA, Conrad SA, Wang H, et al (2011) Resuscitation with balanced electrolyte solution prevents hyperchloremic metabolic acidosis in patients with diabetic ketoacidosis. Am J Emerg Med 29: 670674

29. Gayathri Bhuvaneswaran K, Ramachandran R, Subramanian M et al (2017) Randomized Doubleblind Trial of Ringer Lactate Versus Normal Saline in Pediatric Acute Severe Diarrheal Dehydration. J Pediatr Gastroenterol Nutr 65(6): 621-626

30. Abu-El-Haija M, Kumar S, Quiros JA, et al (2018). Management of Acute Pancreatitis in the Pediatric Population: A Clinical Report From the North American Society for Pediatric Gastroenterology, Hepatology and Nutrition Pancreas Committee. J Pediatr Gastroenterol Nutr 66:159-176

31. Preiser JC, Ichai C, Orban JC, Groeneveld AB (2014) Metabolic response to the stress of critical illness. Br J Anaesth. 113(6):945-954.

32. Marik PE, Bellomo R (2013) Stress hyperglycemia: an essential survival response! Crit Care 17(2):305

33. Sümpelmann R, Mader T, Eich C et al (2010) A novel isotonic-balanced electrolyte solution with $1 \%$ glucose for intraoperative fluid therapy in children: results of a prospective multicentre observational post-authorization safety study (PASS). Paediatr Anaesth 20(11):977-981

34. Marino LV, Valla FV, Beattie RM, Verbruggen SCAT (2020) Micronutrient status during paediatric critical illness: A scoping review. Clin Nutr 39(12):3571-3593

\section{Tables}

Table 1. Characteristics of Survey Respondents 
Role of prescribers:

\begin{tabular}{lll}
\hline Consulting pediatric intensivist (attending) physician & 131 & $85 \%$ \\
\hline PICU fellow/resident/junior & 13 & $8 \%$ \\
\hline Anesthetist & 10 & $7 \%$
\end{tabular}

\section{Iypes of PICU:}

\begin{tabular}{lll}
\hline General PICU only (without Cardiac ICU) & 58 & $38 \%$ \\
\hline Mixed general PICU \& Cardiac ICU & 40 & $26 \%$ \\
\hline Mixed general PICU \& Cardiac ICU \& NICU & 12 & $8 \%$ \\
\hline Mixed general PICU \& NICU (without Cardiac ICU) & 32 & $21 \%$ \\
\hline Cardiac ICU only & 4 & $2 \%$ \\
\hline Specialised PICU e.g. burns/neuroscience & 6 & $4 \%$ \\
\hline Adult ICU (that admits children) & 2 & $1 \%$ \\
\hline
\end{tabular}

Type of Hospital:

\begin{tabular}{lll} 
Specialist Children Hospital & 87 & $57 \%$ \\
\hline Local Hospital & 45 & $29 \%$ \\
\hline University Hospital & 22 & $14 \%$
\end{tabular}

Results are expressed in number and percentage (\%)

PICU: Pediatric Intensive Care Unit, NICU: Neonatal Intensive Care Unit, ICU: Intensive Care Unit.

Table2. Frequency of prescribing IV-MFT in Different clinical conditions. 


\begin{tabular}{|c|c|c|c|c|}
\hline & Always & $\begin{array}{l}\text { Often/ } \\
\text { Sometimes }\end{array}$ & $\begin{array}{l}\text { Rarely/ } \\
\text { Never }\end{array}$ & $\begin{array}{l}\text { Number of respondents/ } \\
\text { questions }\end{array}$ \\
\hline Non-severe bronchiolitis & $3(2 \%)$ & $48(31 \%)$ & $\begin{array}{l}82 \\
(54 \%)\end{array}$ & 153 \\
\hline $\begin{array}{l}\text { Severe diabetic keto } \\
\text { acidosis }\end{array}$ & $\begin{array}{l}128 \\
(84 \%)\end{array}$ & $20(13 \%)$ & $5(3 \%)$ & 153 \\
\hline Post abdominal surgery & $\begin{array}{l}113 \\
(74 \%)\end{array}$ & $36(24 \%)$ & $3(2 \%)$ & 152 \\
\hline $\begin{array}{l}\text { Fasting }<24 \text { h (nill by } \\
\text { mouth) }\end{array}$ & $73(49 \%)$ & $67(45 \%)$ & $10(7 \%)$ & 150 \\
\hline ARDS & $52(34 \%)$ & $76(50 \%)$ & $\begin{array}{l}25 \\
(16 \%)\end{array}$ & 153 \\
\hline
\end{tabular}

Results are expressed in number and percentage (\%)

ARDS: Acute Respiratory Distress Syndrome

Table 3. Type of fluids depending clinical situation 
Cristalloide:

Hypotonic solution

unbalanced
Cristalloide: Cristalloide:

Isotonic

solution

unbalanced
Isotonic

solution
Number of respondents/ questions

\section{viral gastroenteritis not tolerating oral rehydration}

- Serum Na normal : $137 \mathrm{mmol} / \mathrm{L}$

5 months old $10(7 \%)$

$72(47 \%) \quad 71(46 \%) \quad n=153$

patient

12 years old $8(5 \%)$

$70(46 \%)$

$75(49 \%)$

$n=153$

patient

- Serum Na above normal: 149 mmol/L

5 months old $\quad 41(27 \%)$

patient

$36(24 \%) \quad 76(59 \%) \quad n=153$

12 years old

$38(25 \%)$

$35(23 \%)$

$80(52 \%)$

$n=153$

patient

\section{Status epilepticus and still somnolent}

5 months old $6(4 \%)$

patient

$85(56 \%)$

$62(41 \%) \quad n=153$

12 years old $4(3 \%)$ patient

$81(53 \%)$

$68(44 \%)$

$n=153$

\section{Persistent respiratory distress on NIV}

7 days old $\quad 38(25 \%)$

patient

5 months old $20(13 \%)$

patient

12 years old $13(9 \%)$

patient
$59(38 \%)$

$72(47 \%)$

$71(46 \%)$

$69(45 \%)$

$n=153$

\section{ARDS ventilated on PICU (1st 48 h).}

5 months old $14(9 \%)$

$70(46 \%)$

$69(45 \%)$

$n=153$

patient

12 years old $7(5 \%)$

$71(46 \%)$

$75(49 \%)$

$n=153$ patient

\section{Traumatic brain injury ventilated on PICU}

14 years old $2(1 \%)$

$82(55 \%)$

$66(44 \%)$

$n=150$ patient 
Results are expressed in number and percentage (\%)

NIV: Non-Invasive Ventilation, ARDS: Acute Respiratory Distress Syndrome, PICU: Pediatric Intensive Care Unit

Table 4. Frequency of prescribing different nutrients and electrolytes within the fluid

\begin{tabular}{llll} 
& Always & Often/Sometimes & Rarely/Never \\
\hline Glucose & $70(46 \%)$ & $80(52 \%)$ & $3(2 \%)$ \\
\hline Potassium & $38(25 \%)$ & $111(73 \%)$ & $4(3 \%)$ \\
\hline Phosphate & $2(1 \%)$ & $89(59 \%)$ & $60(40 \%)$ \\
\hline Magnesium & $3(2 \%)$ & $81(54 \%)$ & $67(44 \%)$ \\
\hline Calcium & $14(9 \%)$ & $96(63 \%)$ & $43(28 \%)$ \\
\hline Trace Elements & $3(2 \%)$ & $58(38 \%)$ & $90(60 \%)$ \\
\hline Vitamins & $6(4 \%)$ & $57(38 \%)$ & $88(58 \%)$
\end{tabular}

Results are expressed in number and percentage (\%)

\section{Figures}




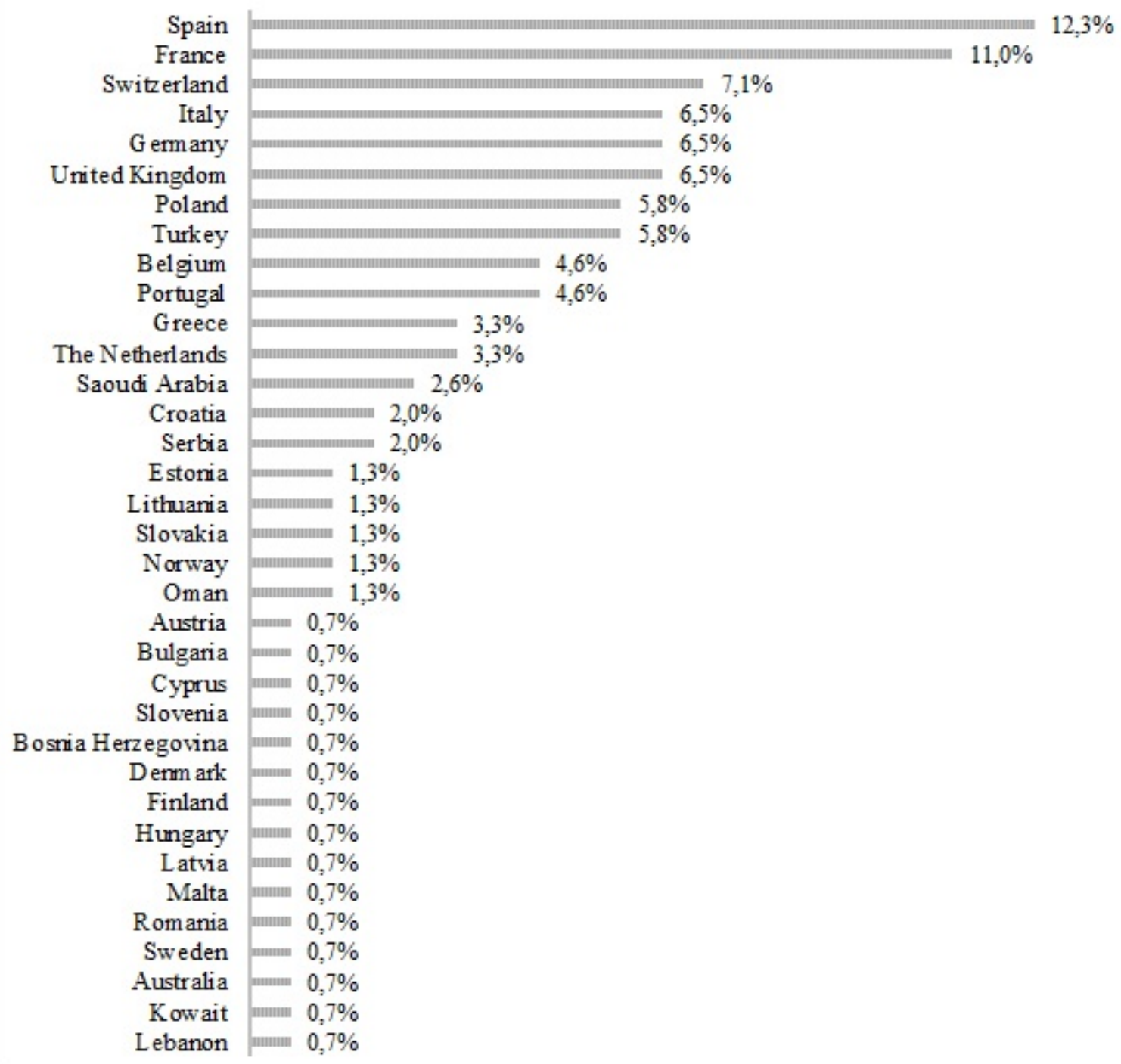

\section{Figure 1}

Geographical distribution of survey respondents

Children in general pediatrics ward

Children on Non invasive ventilation

Children on invasive ventilation

Children with renal failure

Children following neurosurgery

Children following general surgery

Children following cardiac surgery

Children with cardiac conditions 
Figure 2

Strategy applied by the respondents within different clinical conditions in term of amount of IV-MFT

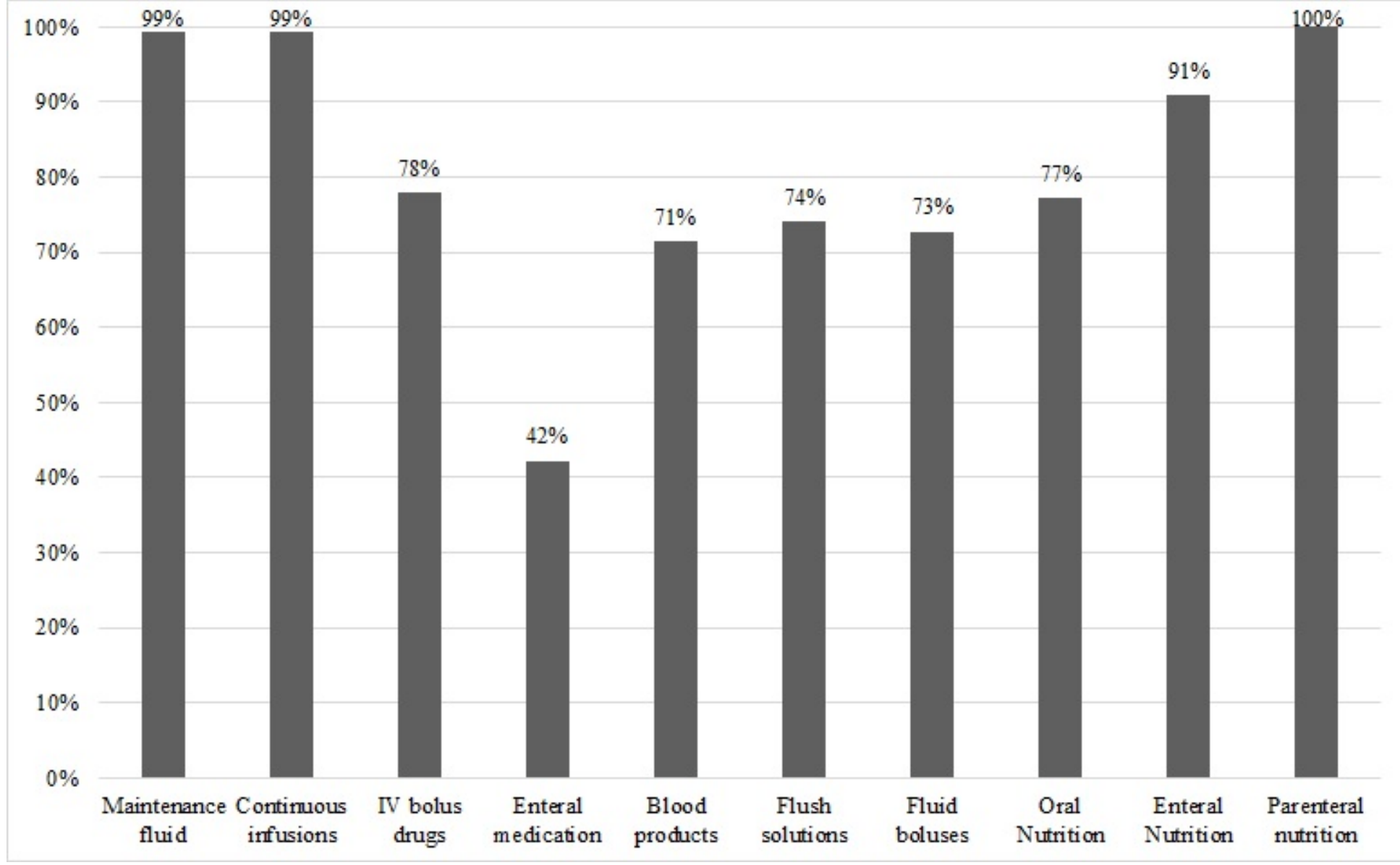

Figure 3

The fluids considered in the total fluid intake by the respondents.

\section{Supplementary Files}

This is a list of supplementary files associated with this preprint. Click to download.

- SDC1.pdf

- SDC2.pdf 